\title{
Impact of Work Schedules on the Sleep Patterns of Railroad Workers using CHAID Neural Network and Ensemble Models of Machine Learning
}

\author{
Devyani Gupta, Nikita Pande, Jitendra Shreemali, Prasun Chakrabarti
}

\begin{abstract}
The study examines the impact of work schedules on the sleep patterns of railroad workers in the USA. The study used the CHAID model, Neural Network and the Ensemble model to identify factors that have a greater impact on sleep patterns. Age, number of children / dependents are found to be key factors for sleep apnea as well as sleep disorders while job pressure and work hours are seen to be the third factor for sleep apnea and sleep disorder respectively. CHAID model provided the highest accuracy (92\%) for sleep disorder while the ensemble model provided an accuracy of over $93 \%$ for sleep apnea.
\end{abstract}

Keywords- Neural Network, CHAID, Ensemble Model, Sleep disorder, Sleep Apnea

\section{INTRODUCTION}

Sleep is a natural process which helps in refreshing one's body and mind, to reduce stress and to maintain overall health of a human body. Humans sleep for almost one third of their lifetime. Everyone has a different sleep pattern. Their sleep pattern is directly or indirectly affected by various factors such as stress, type of work they perform, age, mental and physical health, medical history, etc. Children and adolescents sleep for longer duration as compared to adults. Similarly, young adults sleep more than older people. Most adults are comfortable with 7 to $8 \mathrm{~h}$ daily sleep.

Sleep is a circadian combination of the changes in the biochemical, physiological as well as psychological processes of an individual. Any change in this circadian rhythm or any variation in daily routine of an individual's lifestyle leads to a variety of disorders. Ability to cope up with these disturbances also varies from person to person.

Studying and analyzing variations or activities of individuals is helpful in dealing with certain sleep disorders and to find out the suitability of individuals for various jobs requiring unusual work schedules.

Sleep disorder constitutes a variety of diseases which can cost very much to the society and can have remarkable consequences on the health of individuals.

40 million Americans are suffering from chronic sleep disorders or wakefulness. These diseases affect their work, social life and travelling.

Revised Manuscript Received on March 15, 2020.

Devyani Gupta*, Techno India NJR Institute of Technology, College in Biliya, Rajasthan, India.

Nikita Pande, Techno India NJR Institute of Technology, College in Biliya, Rajasthan, India.

Jitendra Shreemali, Techno India NJR Institute of Technology, College in Biliya, Rajasthan, India.

Prasun Chakrabarti, Techno India NJR Institute of Technology, College in Biliya, Rajasthan, India.

E-mail: devyanigupta0310@gmail.com
There have been more than 39,000 cardiovascular deaths due to sleep deprivation and it costs over 16 billion annually. Indirect costs of another 50 to $\$ 100$ billion is also added because of accidents, property destruction, litigation, hospitalization, and death.

\section{Common Sleep Disorders Encountered}

Sleep disorders are very common in the general population and it is associated with significant medical, psychological, and social disturbances.

Several people report experiencing problems related to their sleep such as inadequate sleep or inadequately rested or the sleep itself not being sound. These sleep disorders can cause multiple problems. For instance, it may lead to low energy levels to carry out required tasks during the daytime, irritability and a sense of being tired affecting the individuals' professional, social and/or personal life. Sometimes the sleep disorder itself may be a symptom or one of the outcomes of a medical condition like sleep apnea or a more serious mental health condition like anxiety/ depression. Sleep issues are sometimes a warning sign that point to very serious medical conditions like bipolar disorder. Sleep related problems can also worsen many medical and mental health conditions along with affecting sleep.

Most of the sleep related problems are categorized as:-

1). Insomnia

2). Excessive sleepiness, or

3). Abnormal behaviour during sleep.

There are so many sleep disorders encountered, but most common sleep disorders are:-

\section{Insomnia}

Insomnia is easily the frequently encountered sleep wakerelated sleep disorder that could be reflective of the patient undergoing psychological disturbances. It is a social health problem of high significance that requires accurate diagnosis and effective treatment.

Insomnia is a condition where the person complains of not getting the required amount of sleep or not feeling energetic after their habitual sleep routine. This disorder is generally seen in women and has a proportion from $9 \%$ to $35 \%$ of women.

Classification of insomnia is done on the basis of severity. This insomnia could be mild, moderate, severe or duration acute, sub acute, chronic. Transient insomnia is triggered due to acute stress, travel, or while attempting to sleep in an unfamiliar environment. The symptoms of transient insomnia are generally resolved once the stress is reduced or removed, or the individual adapts to the stress.

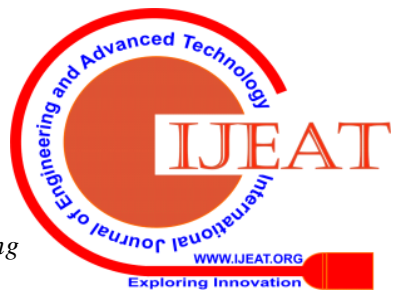


Chronic insomnia can be primary, or secondary symptom of cardiac, environmental, behavioral and many more disorders.

Medical, neurological, environmental, drugs, or psychiatric causes may lead to secondary insomnia. Medical causes for secondary insomnia are heart related diseases, pain, thyroid problems, menstrual related problems, etc.

Workers working in shifts usually suffer from wakefulness, problems in sleeping, poor sleep quality or exorbitant sleepiness.

\section{Sleep Apnea}

Sleep apnea is a potentially serious sleep disorder. In this disorder, breathing repeatedly stops and starts. This disorder is commonly known as snoring. If someone snores loudly and feels tired even after a full night's sleep, the person might have sleep apnea.

Apnea is broadly explained as a disturbance in the flow of air for more than $10 \mathrm{sec}$. Hypopnea is an abnormal respiratory event lasting longer than $10 \mathrm{~s}$ associated with at least a $30 \%$ reduction in thoraco-abdominal movement or airflow compared to baseline, associated with $\geq 4 \%$ oxygen desaturation.

\section{Parasomnia: Sleepwalking, Night Terrors and Nightmares}

The parasomnias are very common in people of every age group. About $15 \%$ of children have one or more sleepwalking problems, while 1-3\% suffer from night terrors or nightmares. About $10 \%$ of the population is suffering from the problem of nightmares.

Parasomnia refers to abnormal behaviour during sleeping or abnormal physical activities. Most common form of parasomnia includes disorders of arousal, partial arousal, and sleep-stage transition.

Parasomnia sometimes occurs due to heredity in the family. Other factors for parasomnia include fragmentation of sleep, pain, changes in surroundings, migraine headaches, etc.

\section{LITERATURE SURVEY}

Worley (2018) reports it does not matter how hectic our lives become, we can no longer allow oneself to ignore the saying of various researches about the importance of sleep in one's life for their safety and well-being. In the past few decades, chaotic lifestyles, hectic work schedules and improper sleep patterns have become a threat to both safety and health. Alharbi AAM, Alotaibi TM, Almalki AM, Althekri MSH, Alshadokhi OA, et al. (2017) states that sleep deficiency limits the ability of a person to solve the problems effectively and to think, in other words individuals who work for longer time durations lack the efficiency to learn effectively. Sleep also affects the accuracy of making decisions and interpreting situations. Lack of sleep can lead to swelling of eyes and pale skin. In the report Harma M. , Sallinen, Ranta, Mutanen, Muller (2002) says that $50 \%$ of the traffic controllers and train drivers during their night shifts experience severe fatigue. During night shifts, work performance impairment because of fatigue is reported by $21-37 \%$ of train drivers and $13-19 \%$ of traffic controllers. Train drivers and traffic controllers of younger ages (under 43 years) experience more fatigue during daytime, while performance of older ones decreases during night shifts. Jillian Dorrian, Stuart D. Baulk, Drew Dawson (2010) reported that fatigue-related disorders or problems and error in work can occur because of sleeping for less than $5 \mathrm{~h}$ before work and/or working continuously for more than 16 h. Safety declines if a worker works for more than 8 hours and the probability for accident roughly doubles if working hours are extended to 10 hours.

Owens J. (2014) says that deficiency of sleep in adolescents has a serious risk on academic success, emotional and physical health as well as safety of youth. If it continues it may lead to higher risks. Velayudhan Mohan Kumar (2014) reported that sleep patterns have a high impact on an individual. Changes in quantity, pattern and quality of sleep results in sleep disorders. The sleep disorder can further affect the individual's health if interruptions to sleep are persistent and repeated. If undiagnosed, then sleep complaints will lead to socio-economic consequences and misery to sufferers. Sleepiness during working hours may be due to economic, environmental or any social reasons. Kohyama J., et. al. (2018) says that insufficient sleep results in brain dysfunction. It also leads to cognitive, behavioural and physical problems. Sleep deprivation sometimes leads to obesity and metabolic syndrome.

\section{DATA AND ANALYSIS METHODOLOGY}

The data set used included over 440 data points including data on total years as dispatcher, marital status, number of children or dependents, usage of caffeinated drinks, health status, average hours of work per week and whether the person suffers from sleep disorder or apnea. It also examines other likely possibilities like persons feeling mentally drained and total number of life events. Modeling using machine learning algorithms

was carried out for sleep apnea as well as sleep disorder. Data was taken from www.kaggle.com/nairaminasyan/sleep-patterns . The models used included the neural network, the ensemble model and Chi-squared Automatic Interaction Detection model. The IBM SPSS Modeler tool was used to run the models. Findings/output of these models is presented below: Neural Networks: The neural network algorithm is inspired by the neural network present in animal brains. It consists of an input layer which comprises of all the features or properties observed in the input data, an output layer which identifies the cluster to which the input data belongs. Between the input and output layers, there are hidden layers present. Number of hidden layers depends on the requirements. In these layers neurons compute the weighted inputs to produce output for the next layer with the help of some activation function and biases. Neural network creates a layered directed weighted graph.

Ensemble Model: The ensemble model is a type of supervised learning algorithm. It is a hybrid model, in other words, two or more models are combined together to make predictions and to produce desired output from the given input data. This approach is used to minimize the prediction error in the final output. Instead of running various models on the same dataset, it is significant to use a combination of those models to reduce time and complexity. 
Chi-squared Automatic Interaction Detection model: The CHAID is a classification method that uses the chi-square statistics to build decision trees for identifying optimal splits. This is done, first by examining cross-tabulations between all input fields and the outcome based on the level of significance (based on the chi-square statistic). When multiple inputs are found to be statistically significant, CHAIN carries out an ordering based on the parameters (input) that has the highest significance (or the smallest $p$ value).

The figure below gives a schematic of the IBM SPSS Modeler screen for the three models used in both cases, namely, Neural Networks, CHAID and Ensemble model.

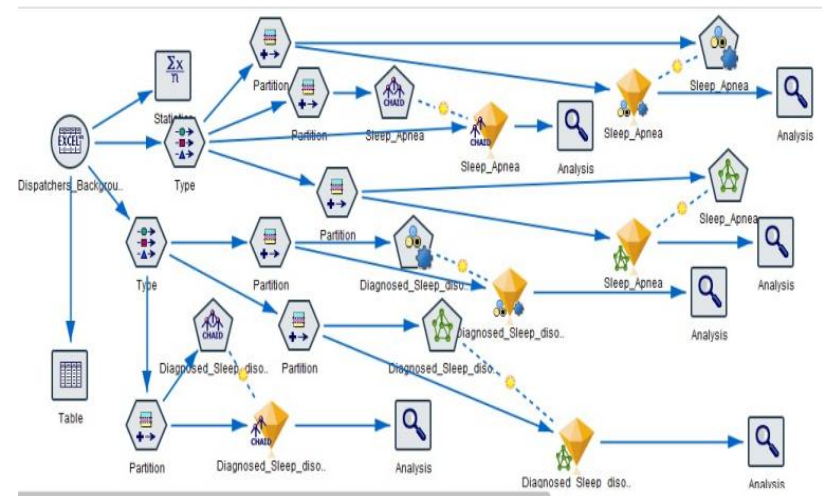

Fig 1: Models: IBM SPSS Modeler

\section{CONCLUSION AND FINDINGS}

The findings are briefly presented below:

1. Classification using the Neural network shows over $90 \%$ correctly classified cases of Sleep Apnea for the test data;

Table 1: Neural Network Classification

\begin{tabular}{|l|r|r|r|r|}
\hline 'Partition' & 1_Training & 2_Testing & \\
\hline Correct & 289 & $89.75 \%$ & 111 & $90.24 \%$ \\
\hline Wrong & 33 & $10.25 \%$ & 12 & $9.76 \%$ \\
\hline Total & 322 & & 123 & \\
\hline
\end{tabular}

The neural network used is presented below:

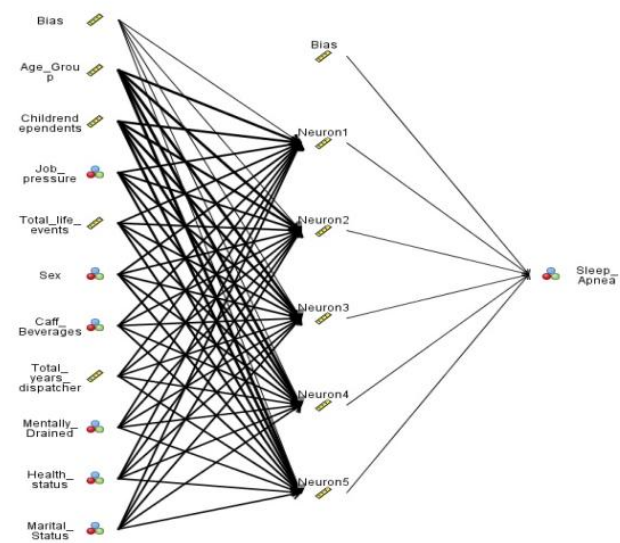

Fig 2: Neural Network Layers

The predictor importance based on the neural network above is given below:

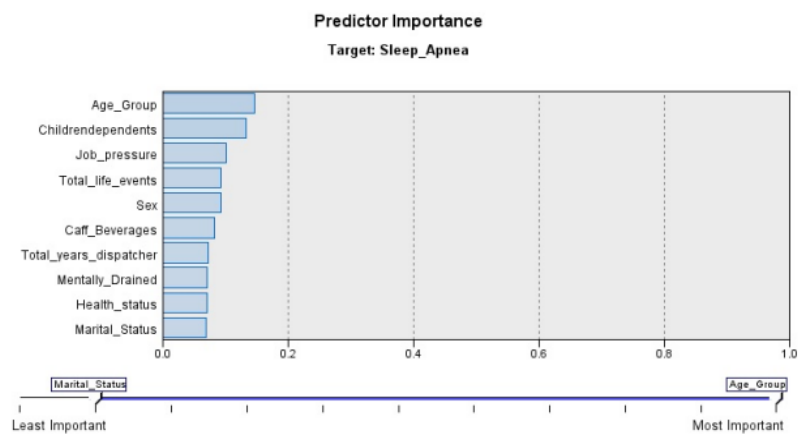

Fig 3: Predictor Importance

Age (0.15), Number of children/dependents (0.13) and Job Pressure (0.1) are the most important factors that enhance chances of sleep apnea.

2. The CHAID model provides over $92 \%$ correct classification of the Sleep Apnea data

Table 2: CHAID Classification

\begin{tabular}{|l|r|r|}
\hline Correct & 410 & $92.13 \%$ \\
\hline Wrong & 35 & $7.87 \%$ \\
\hline Total & 445 & \\
\hline
\end{tabular}

3. Ensemble auto-classification of Sleep Apnea provides the best results with over 93\% correctly classified cases of Sleep Apnea for the test data. Table 3 : Ensemble Classification

\begin{tabular}{|l|r|r|r|r|}
\hline 'Partition' & 1_Training & & 2_Testing & \\
\hline Correct & 295 & $91.61 \%$ & 115 & $93.5 \%$ \\
\hline Wrong & 27 & $8.39 \%$ & 8 & $6.5 \%$ \\
\hline Total & 322 & & 123 & \\
\hline
\end{tabular}

4. Classification using the Neural network shows over $85 \%$ correctly classified cases of Sleep Disorder for the test data;

Table 4: Neural Network Classification

\begin{tabular}{|l|r|r|r|r|}
\hline 'Partition' & 1_Training & 2_Testing & \\
\hline Correct & 280 & $86.96 \%$ & 105 & $85.37 \%$ \\
\hline Wrong & 42 & $13.04 \%$ & 18 & $14.63 \%$ \\
\hline Total & 322 & & 123 & \\
\hline
\end{tabular}

The schematic of Neural Network used is given below:

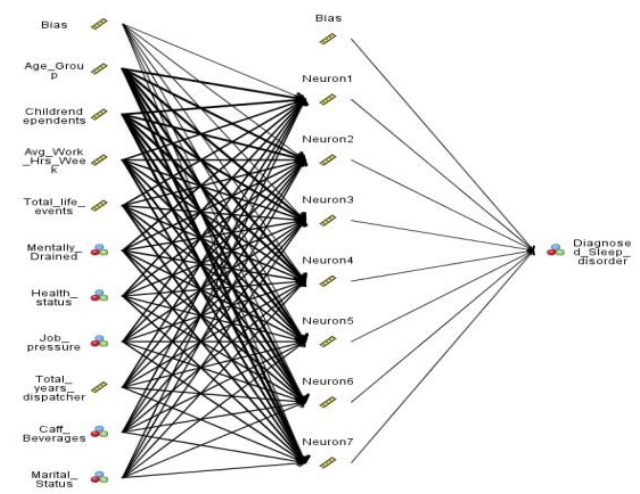

Fig 4: Neural Network Layers 
The predictor importance based on the neural network above is given below:

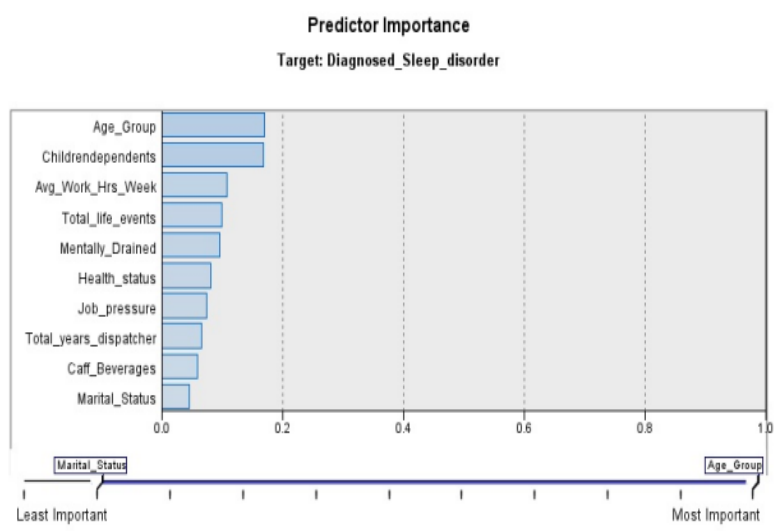

Fig 5: Predictor Importance

Age (0.17), Number of children/dependents (0.17) and Average Hours of work (0.11) are the most important factors that enhance chances of sleep disorder.

5. Even for Sleep Disorders, the CHAID model provides over $92 \%$ correct classification of the data

Table 5: CHAID Classification

\begin{tabular}{|l|r|r|r|r|}
\hline 'Partition' & 1_Training & 2_Testing & \\
\hline Correct & 286 & $88.82 \%$ & 114 & $92.68 \%$ \\
\hline Wrong & 36 & $11.18 \%$ & 9 & $7.32 \%$ \\
\hline Total & 322 & & 123 & \\
\hline
\end{tabular}

6. Ensemble auto-classification of Sleep Disorder over $83 \%$ correctly classified cases of Sleep Disorder for the test data.

Table 6 : Ensemble Classification

\begin{tabular}{|l|r|r|r|r|}
\hline 'Partition' & 1_Training & & 2_Testing & \\
\hline Correct & 300 & $93.17 \%$ & 103 & $83.74 \%$ \\
\hline Wrong & 22 & $6.83 \%$ & 20 & $16.26 \%$ \\
\hline Total & 322 & & 123 & \\
\hline
\end{tabular}

It can be seen that it is possible to achieve over $90 \%$ accuracy for sleep apnea as well as sleep disorder.

\section{LIMITATION OF STUDY}

The data set used is limited to railroad workers in the USA and does not include other trades. This represents an opportunity for further study by comparing these results with those relating to other trades and also across multiple geographical regions.

\section{REFERENCES}

1. Worley, S.L. (2018) "The Extraordinary Importance of Sleep: The Detrimental Effects of Inadequate Sleep on Health and Public Safety Drive an Explosion of Sleep Research". Pharmacy and Therapeutics, 43(12), pp 758-763, Dec. 2018. Retrieved from www.ncbi.nlm.nih.gov/pmc/articles/PMC6281147/

2. Alharbi AAM, Alotaibi TM, Almalki AM, Althekri MSH, Alshadokhi OA, et al. (2017) "Sleep Disorders and its Effect on Community". Health Sci J. 2017, 11:4. Retrieved from

2. www.hsj.gr/medicine/sleep-disorders-and-its-effect-oncommunity.php?aid=20312

3. Harma M., Sallinen, Ranta, Mutanen, Muller (2002) "The Effect of an Irregular Shift System on Sleepiness at Work in Train Drivers and Railway Traffic Controllers". Journal of Sleep Research, vol. 11, issue 2, pp 141-151, June. 2002. Retrieved from
4. onlinelibrary.wiley.com/doi/full/10.1046/j.1365-2869.2002.00294.x

5. Dorrian J., Baulk S. D., Dawson D. (2010) "Work hours, Workload, Sleep and Fatigue in Australian Rail Industry Employees". Applied Ergonomics, vol. 42, issue 2, pp 202-209, June,2011. Retrieved from www.sciencedirect.com/science/article/abs/pii/S0003687010000864

6. Owens J. (2014) "Insufficient Sleep in Adolescents and Young Adults: An Update on Causes and Consequences". Pediatrics, 134 (3) e921-e932, September 2014. Retrieved from pediatrics.aappublications.org/content/134/3/e921.full\#sec-9

7. Kumar V. M. (2014) "Sleep and Sleep Disorders". Indian J Chest Dis Allied Sci 2008; 50: 129-135. Retrieved from www.researchgate.net/profile/Velayudhan_Kumar/publication/52414 69_Sleep_and_sleep_disorders/links/00b4953b62a1fcc5f8000000/Sle ep-and-sleep-disorders.pdf

8. Kohyama J., Anzai Y., Ono M., Kishino A., Tamanuki K., Takada K., Inoue K., Horiuchi M., Hatai Y. (2018) "Insufficient sleep syndrome: An unrecognized but important clinical entity". Pediatrics Internationals, vol. 60, issue 4, pp 372-375, April 2018. Retrieved from

9. onlinelibrary.wiley.com/doi/abs/10.1111/ped.13519

10. McCrae C. S. and Lichstein K. L. (2001) "Secondary insomnia: diagnostic challenges and intervention opportunities" Sleep Medicine Reviews, Vol. 5, No. 1, pp 47-61, 2001. Retrived from www.med.upenn.edu/cbti/assets/user-

content/documents/LichsteinSIreview.pdf

11. Abad V. C. and Guilleminault C. (2003) "Diagnosis and treatment of sleep disorders: a brief review for clinicians" Dialogues Clin Neurosci. 2003 Dec; 5(4): 371-388 .Retrived from www.ncbi.nlm.nih.gov/pmc/articles/PMC3181779/

12. Vgontzas A. N. and Kales A. (1999) "Sleep and Its Disorders". Annu. Rev. Med. 1999. 50:387-400. Retrieved from m.recoveryonpurpose.com/upload/Sleep\%20and\%20its\%20Disorders. pdf

13. National Alliance on Medical Illness. Retrived from www.nami.org/Learn-More/Mental-Health-Conditions/RelatedConditions/Sleep-Disorders

\section{AUTHORS PROFILE}

Devyani Gupta, is graduating in B.TECH (CSE) from Techno India NJR Institute of Technology, Udaipur. Her areas of interest are data analytics, machine learning, programming and python. She has good

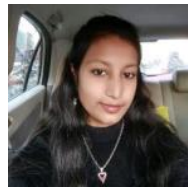
programming skills in $\mathrm{C}, \mathrm{C}++$, Python and Java. She has been a team worker, responsible and confident person and a sincere colleague. She has been an extraordinary student in academics and has volunteered in many events. She is responsible, punctual and creative. She has participated in SIH software and hardware for two consecutive years - SIH software'18 and SIH hardware'19. Worked on city air pollution tracking project as an analyst.

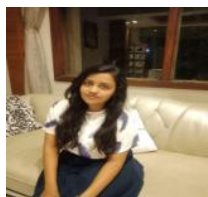

Nikita Pande, is graduating in B.TECH (CSE) from Techno India NJR Institute of Technology Udaipur. Her areas of interest are cloud computing, machine learning, python and the internet of things. She has good programming skills in $\mathrm{C}, \mathrm{C}++$, Python and $\mathrm{R}$. She has been an absolute all rounder in her college time. She is responsible, punctual and creative. She has good leadership qualities and has been a responsible colleague. She has participated in SIH software and hardware for two years - SIH software'20 and SIH hardware'18. Worked on city air pollution tracking project as an analyst and developer.

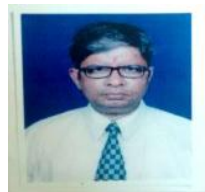

Prof Jitendra Shreemali, is a graduate from IIT Madras with post graduate from IIM Bangalore. He is working as Professor of the Department of Computer Science and Engineering of Techno India NJR Institute of Technology Udaipur. He has worked in reputed companies in India \& abroad for about a decade and half followed by about two decades of academic/research/training experience. He has taught a very wide variety of subjects/courses including operations management, research methodology, and data science besides others. His areas of work include data science, optimization, mathematical modeling and machine learning.

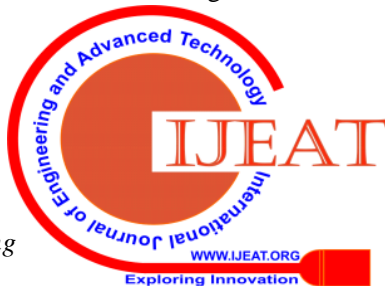




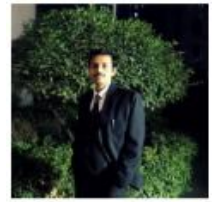

Prasun Chakrabarti, received his $\mathrm{PhD}$ (Engg) from Jadavpur University in 2009. He is working as Executive Dean (Research and International Linkage) and Institute Distinguished Senior Chair Professor, Techno India NJR Institute of Technology. He has several publications, books and 31 filed Indian patents in his credit. He

has supervised ten $\mathrm{PhD}$ candidates successfully. On various research assignments, he has visited Waseda University Japan (2012 availing prestigious INSA-CICS travel grant), University of Mauritius (2015), Nanyang Technological University Singapore $(2015,2016,2019)$, Lincoln University College Malaysia (2018), National University of Singapore (2019), Asian Institute of Technology Bangkok Thailand (2019) and ISI Delhi (2019). He is a Fellow of IETE, ISRD(UK), IAER(London), AE(I), CET(I) and Senior member of the IEEE(USA). FIET 